\title{
A multidisciplinary clinical treatment of locally advanced rectal cancer complicated with rectovesical fistula: a case report
}

\author{
Tiancheng Zhan ${ }^{1}$, Lin Wang ${ }^{1}$, Ming Li ${ }^{1}$, Zhongwu $\mathrm{Li}^{2}$, Yong $\mathrm{Cai}^{3}$, Lin Shen ${ }^{4}$ and Jin Gu${ }^{1 *}$
}

\begin{abstract}
Introduction: Rectal cancer with rectovesical fistula is a rare and difficult to treat entity. Here, we describe a case of rectal cancer with rectovesical fistula successfully managed by multimodality treatment. To the best of our knowledge, this is the first such case report in the literature.

Case presentation: A 51-year-old Chinese man was diagnosed as having rectal cancer accompanied by rectovesical fistula. He underwent treatment with neoadjuvant radiochemotherapy combined with total pelvic excision and adjuvant chemotherapy, as recommended by a multimodality treatment team. Post-operative pathology confirmed the achievement of pathological complete response.
\end{abstract}

Conclusions: This case suggests that a proactive multidisciplinary treatment is needed to achieve complete cure of locally advanced rectal cancer even in the presence of rectovesical fistula.

\section{Introduction}

Most colorectal cancers in the upper rectum or sigmoid colon invade the top of the bladder, and they are clinically treated with a relatively simple en-bloc resection of the invaded bladder [1]. When the tumor is located in the anterior wall in the middle of the rectum it is likely to invade the bladder trigone [1], and when tumor is in the low rectum it is likely to invade the prostate and seminal vesicles. Thus, special handling is required for mid to low rectal cancer, especially in men.

\section{Case presentation}

A 51-year-old Asian man presented to our facility with issues of increased stool frequency (six to eight times a day) accompanied with tenesmus for more than a month. The stool was shapeless and occasionally mixed with small amounts of blood. He was admitted due to worsening of these symptoms and fecaluria accompanied with fever and severe body weight loss.

\footnotetext{
* Correspondence: zlguj@yahoo.com.cn

'Key Laboratory of Carcinogenesis and Translational Research (Ministry of Education), Department of Colorectal Surgery, Peking University School of Oncology, Beijing Cancer Hospital, 52 Fu Cheng Lu, Beijing, Haidian District 100142, PR China

Full list of author information is available at the end of the article
}

A protruding peri-rectal lump in the anterior wall with an uneven surface was found by digital rectal examination. A colonoscopy examination revealed a mucosal bulge $4 \mathrm{~cm}$ from the anal verge with surface erosion and stenosis. A rectal biopsy confirmed the diagnosis of moderately differentiated adenocarcinoma. Pelvic computed tomography $(\mathrm{CT})$ showed a rectal cancer that had invaded the bladder. Cystoscopy revealed a $6 \times 6 \mathrm{~cm}$ lump at the six o'clock position of the bladder neck and trigone with ulcerative erosion on the surface, suggesting an invasion of rectal tumor. Pelvic magnetic resonance imaging (MRI) showed extensive thickening of the wall in the upper rectum with a broken outer membrane by the lesion on the right side that spread to the bladder, where intestinal contents were observed (Figure 1). Multiple enlarged lymph nodes were observed next to iliac vessels inside the mesorectum (Figure 2). The results of a urine white blood cell test were positive. A chest X-ray and abdominal ultrasound did not show any distant metastases. Our patient was clinically diagnosed as having locally advanced rectal cancer, T4bN2M0, stage IIIC, complicated with rectovesical fistula, incomplete rectal obstruction, lower gastrointestinal bleeding, and pelvic local infection.

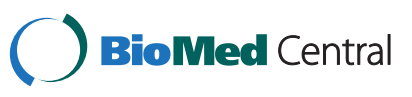




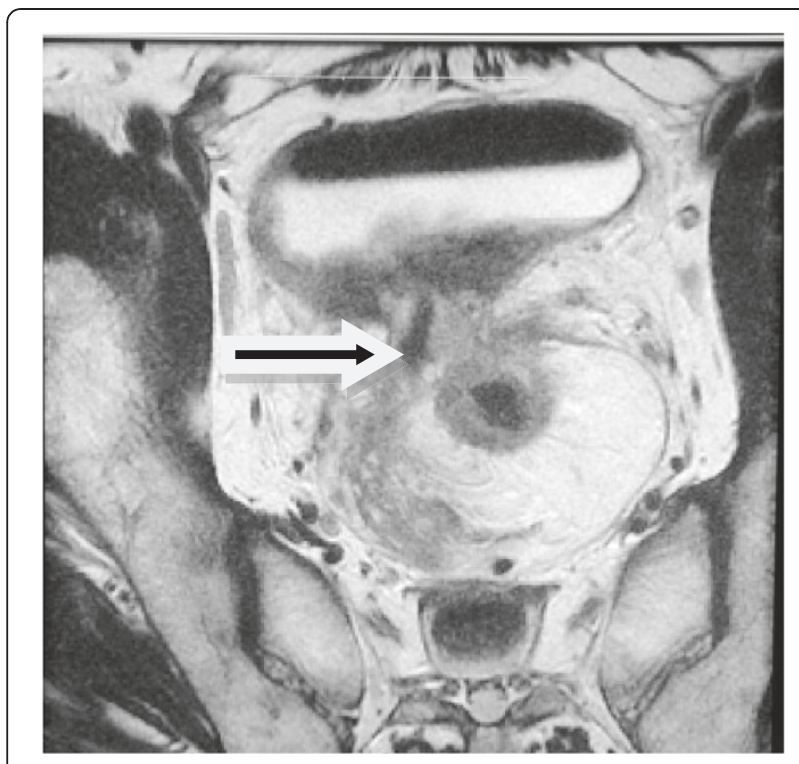

Figure 1 Magnetic resonance image of rectal cancer prior to neoadjuvant therapy. Arrow indicates the rectovesical fistula sinus.

Given that our patient had incomplete intestinal obstruction, rectal bladder fistula, local hemorrhage and infection, radical resection of the tumor was not suitable. A multimodality treatment team consisting of surgeons, physicians, imaging scientists, pathologists and radiologists suggested conducting a transverse colostomy to bypass feces first, subsequently performing pre-operative neoadjuvant therapy when our patient became stable. A week after transverse colostomy, all his clinical symptoms

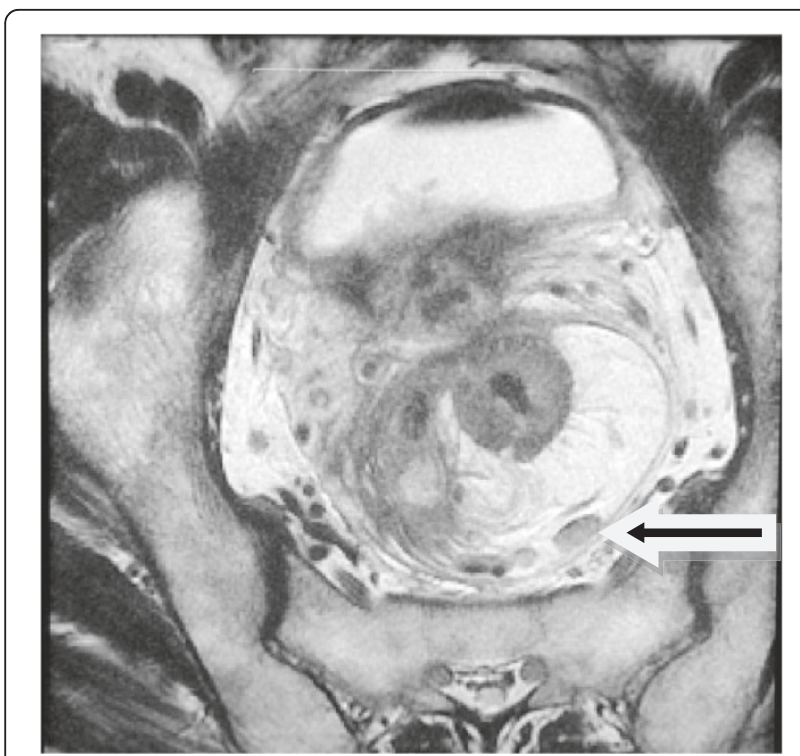

Figure 2 Magnetic resonance image of rectal cancer prior to neoadjuvant therapy. Arrow indicates the enlarged lymph nodes in mesorectum. disappeared and neoadjuvant chemotherapy was administrated using oxaliplatin $80 \mathrm{mg}$ once a week $\left(50 \mathrm{mg} / \mathrm{m}^{2}\right)$ and capecitabine $1.5 \mathrm{~g}$ twice a day $\left(1000 \mathrm{mg} / \mathrm{m}^{2}\right)$ for four weeks, combined with $10 \mathrm{MV} \mathrm{X}$-ray intensity modulated radiation therapy (IMRT) of gross tumor volume (GTV) 50.6Gy/clinical target volume (CTV) 41.8Gy for 22 days.

Two months after neoadjuvant therapy, MRI reassessment showed that the thickness of the upper rectum wall was reduced with significant tumor reduction. The lower end of the tumor was located $4 \mathrm{~cm}$ above the junction of the levator ani muscle and rectum with local invasion to the muscular layer of the wall. Inside the mesorectum, the size of the multiple enlarged lymph nodes next to iliac vessels had also reduced (Figure 3). The lump size localized on the right mesorectal fascia was significantly reduced. However, the irregular thickening of the posterior wall of the bladder was still observed (Figure 4). Positron emission tomographycomputed tomography (PET-CT) confirmed a rectumsigmoid junction cancer spreading to the bladder trigone with metastasis to local lymph nodes of the mesorectum, retroperitoneal and iliac vascular region. No distant metastasis was detected.

With clinical improvement of the rectovesical fistula after neoadjuvant therapy, a total pelvic exenteration (TPE) plus cutaneous ureterostomy was performed for better treatment of the invaded bladder and involved pelvic lymph nodes. Pathological analysis of the surgical specimens (Figures 5, 6, 7) revealed no residual cancer cells, lymphovascular invasion (LVI) or local lymph nodes metastasis (out of 22 lymph nodes), suggesting a

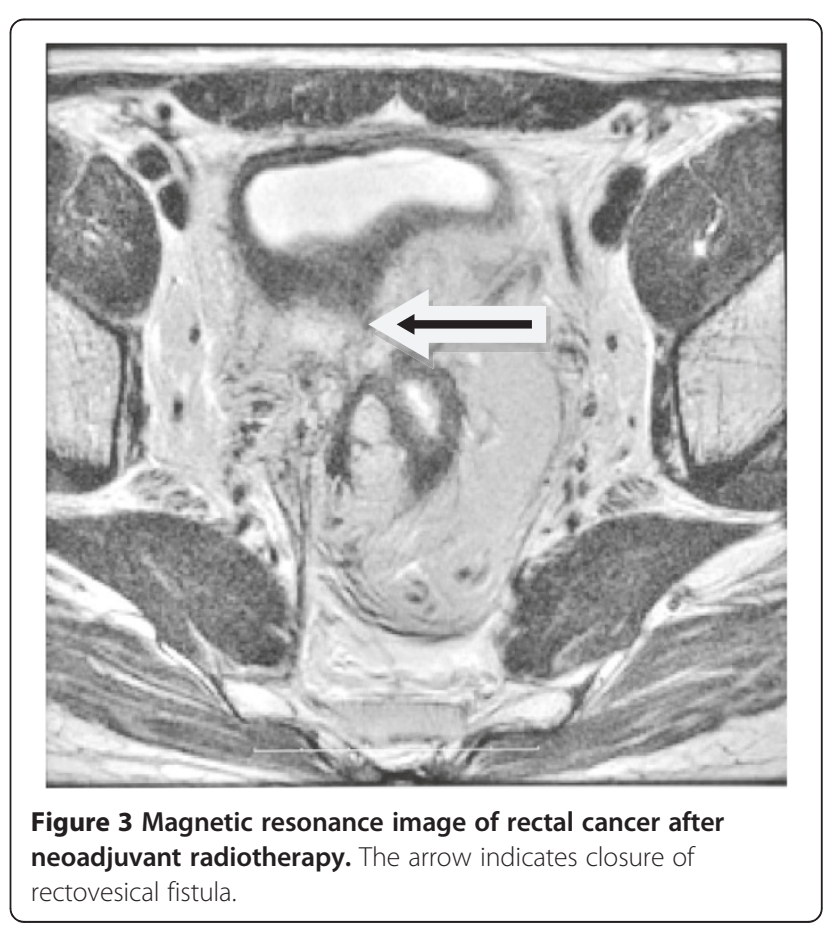




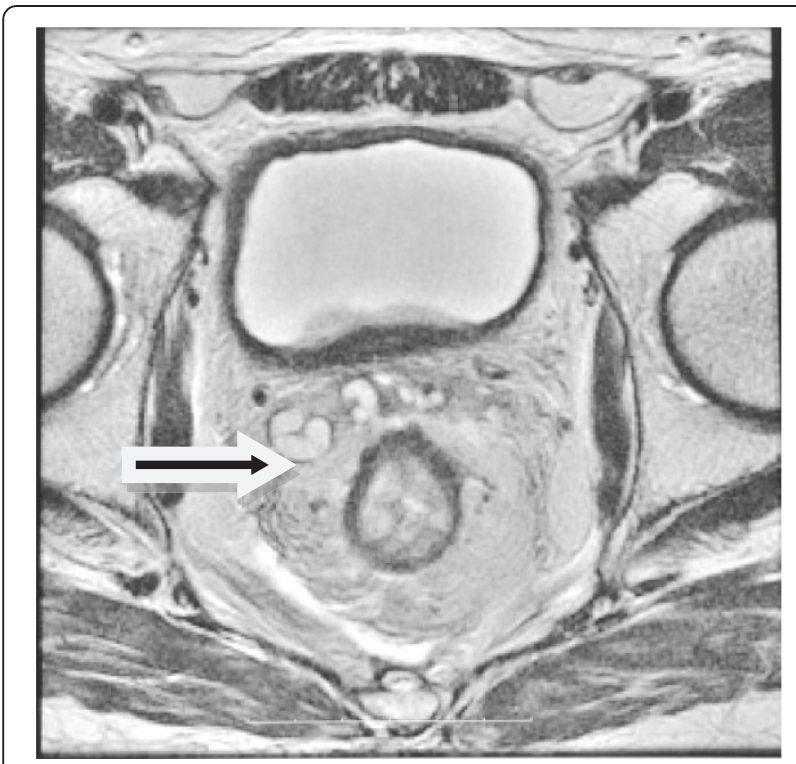

Figure 4 Magnetic resonance image of rectal cancer after neoadjuvant radiotherapy. The arrow indicates the reduced tumors. Some enlarged lymph nodes have disappeared.

pathological complete response (PCR) after neoadjuvant therapy (Figure 8). Five cycles of XELOX regimen (capecitabine plus oxaliplatin) were given as adjuvant therapy. One month after TPE, no abnormal enhancement sites and enlargement of lymph nodes were found in the pelvis by MRI (Figure 9).

\section{Discussion}

According to the National Comprehensive Cancer Network (NCCN) guidelines, neoadjuvant therapy should be

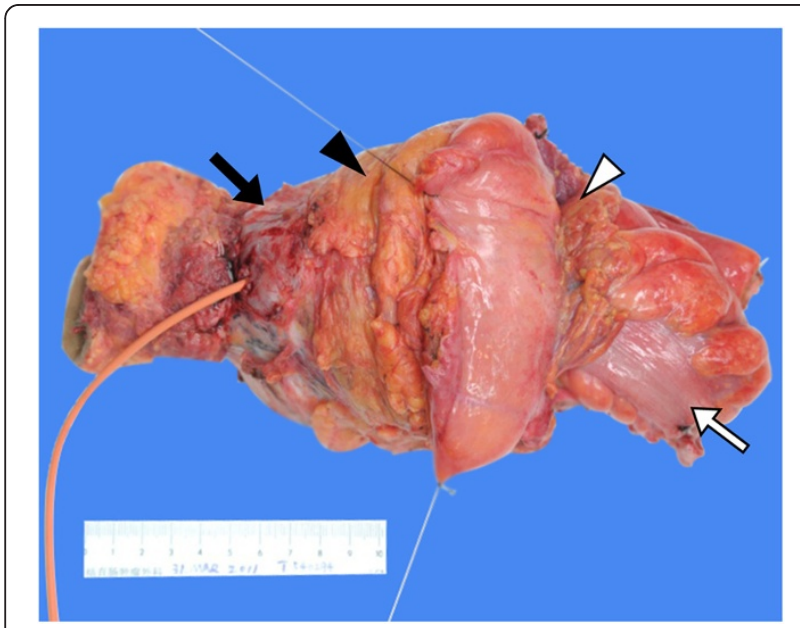

Figure 5 Specimen of total pelvic exenteration surgery sutured at the peritoneal reflection. $\triangleright$ Indicates the peritoneal reflection, - indicates the mesorectal region, $\Rightarrow$ indicates the rectal region, $\Rightarrow$ indicates the invasion of rectal cancer through the bladder wall.

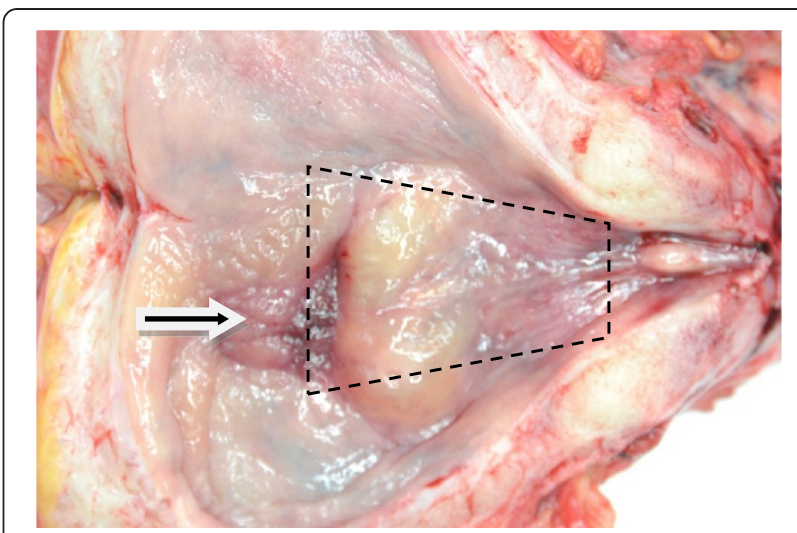

Figure 6 Specimen of total pelvic exenteration surgery showing the triangle area of bladder. The arrow indicates the invasion of rectal cancer through the bladder wall.

given for the treatment of locally advanced rectal cancer (LARC) [2]. However, for tumors that invade into the bladder trigone resulting in rectovesical fistula, the treatment becomes complicated. Therefore, a multidisciplinary clinical team is needed to find comprehensive solutions [3]. All the symptoms such as fever, blood in the stool, fecaluria, and incomplete intestinal obstruction present in our patient resulted from the bladder fistula caused by local invasion of rectal cancer. After under going the operation to create a diverting stoma, all his symptoms disappeared within a week, which helped our patient undergo further treatment.

The incidence of fecaluria caused by rectovesical fistula is relatively low in rectal cancer invading the bladder. Since simple rectal resection would not remove residual tumor in the bladder, we administered neoadjuvant

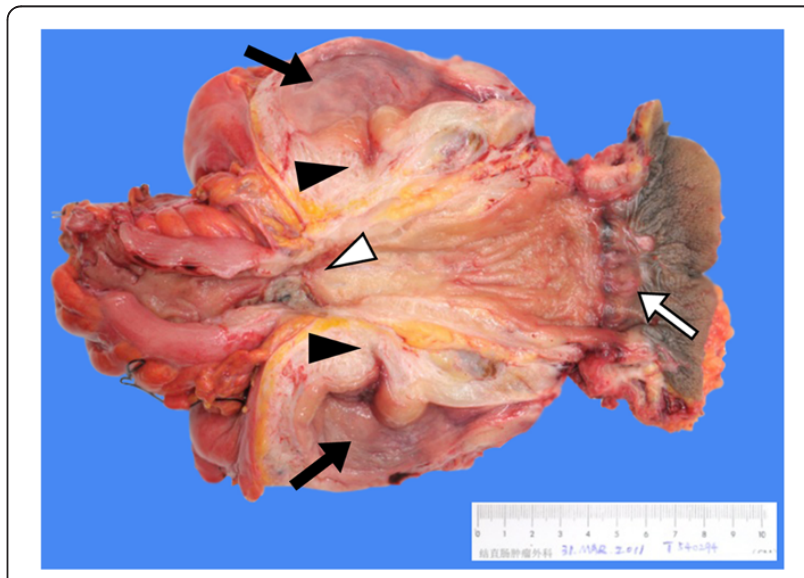

Figure 7 Profile view of total pelvic exenteration surgery specimen. $\triangleright$ Indicates the necrotic rectal cancer following chemoradiotherapy, $\boldsymbol{*}$ indicates the closed rectovesical fistula, $\Rightarrow$ indicates the anal region, $\boldsymbol{m}$ indicates the bladder mucosa. 


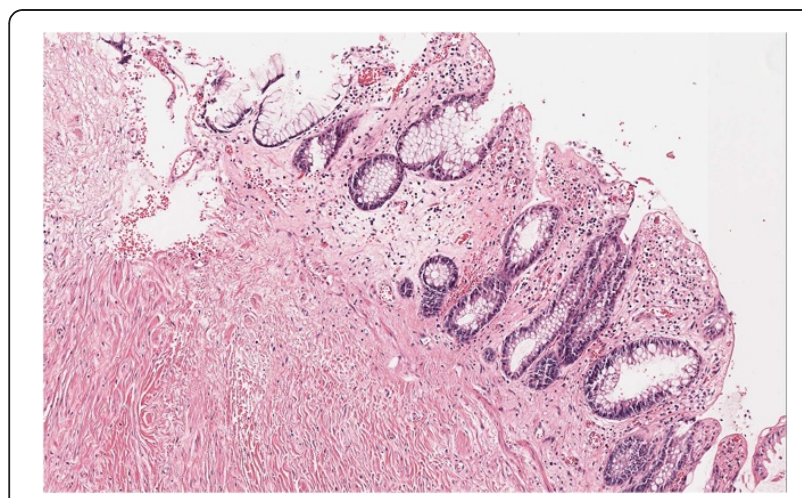

Figure 8 Pathology of resected specimen. Hematoxylin and eosin stain, $\times 100$.

chemotherapy using a XELOX regimen combined with $50.6 \mathrm{~Gy}$ of pre-operative radiation $[4,5]$. The standard preoperative treatment for locally invasive rectal cancer in our hospital included fractional radiation with cumulative 50.4Gy combined with oral administration of capecitabine. In addition to the standard pre-operative treatment, four weeks of oxaliplatin was also given in this case to enhance tumor killing and increase the sensitivity of radiation therapy. This treatment may also contribute to the control of distant metastasis caused by long-term pre-operative treatment. Reassessment after neoadjuvant therapy showed tumor shrinkage and absence of distant metastases. A reasonable therapeutic option could be the performance of a

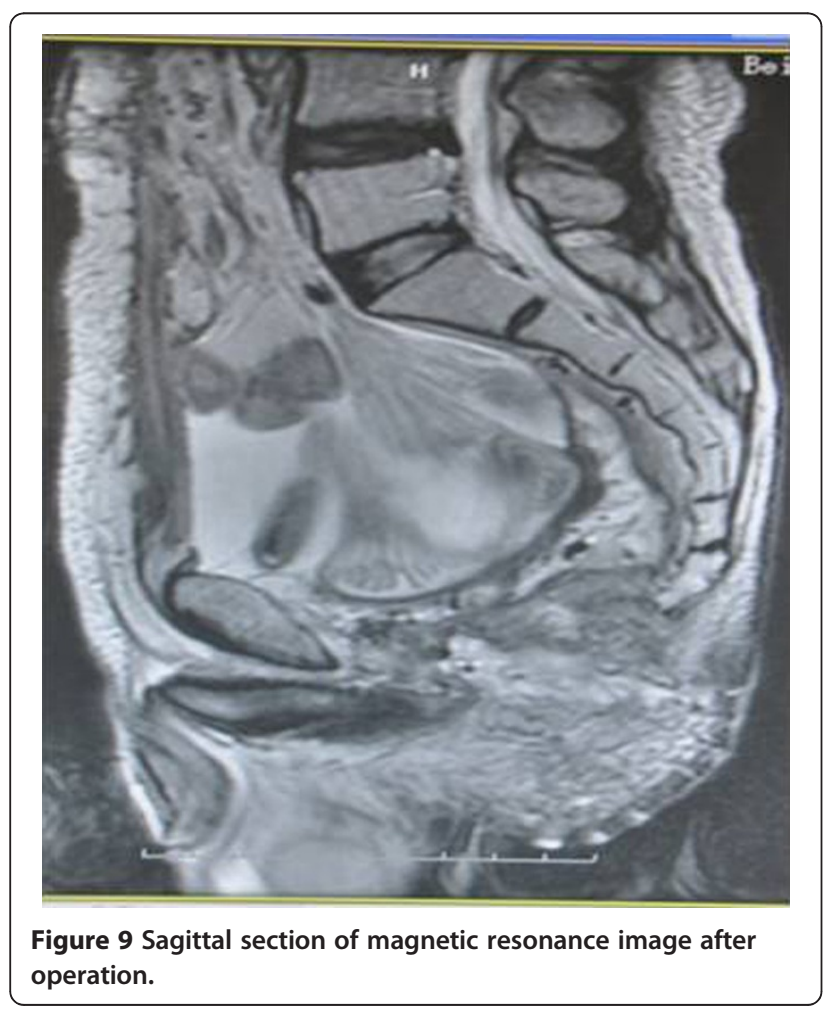

limited surgical procedure if cystoscopy excludes residual tumor in the bladder. Then, a strict urologic follow-up could show eventual vesical relapse and a secondary total cystectomy could be performed. However, we believe that pathological analysis is more accurate than cystoscopy for the diagnosis of residual tumor in the bladder. Considering the multiple enlarged lymph nodes and the good general condition of our patient to tolerate the operation, we performed TPE. Post-operative adjuvant therapy was carried out based on the opinion that post-operative adjuvant therapy is necessary even for a PCR of rectal cancer $[2,4,5]$.

\section{Conclusions}

The findings from our patient's case suggest that a proactive multidisciplinary treatment is needed to achieve complete cure for locally advanced rectal cancer complicated with rectovesical fistula.

\section{Consent}

Written informed consent was obtained from the patient for publication of this manuscript and any accompanying images. A copy of the written consent is available for review by the Editor-in-Chief of this journal.

\section{Competing interests}

The authors declare that they have no competing interests.

\section{Authors' contributions}

TZ analyzed and interpreted the data from our patient regarding rectal cancer and the rectovesical fistula, and was a major contributor in writing the manuscript. LW took the picture for the specimen. ZL performed histological examination of the specimen. JG, ML and TZ performed the TPE for our patient. LS designed the chemotherapy regimen and YC designed the radiotherapy regimen for our patient. All authors read and approved the final manuscript.

\section{Author details}

${ }^{1}$ Key Laboratory of Carcinogenesis and Translational Research (Ministry of Education), Department of Colorectal Surgery, Peking University School of Oncology, Beijing Cancer Hospital, 52 Fu Cheng Lu, Beijing, Haidian District 100142, PR China. ${ }^{2}$ Key Laboratory of Carcinogenesis and Translational Research (Ministry of Education), Department of Pathology, Peking University School of Oncology, Beijing Cancer Hospital, 52 Fu Cheng Lu, Beijing, Haidian District 100142, PR China. ${ }^{3}$ Key Laboratory of Carcinogenesis and Translational Research (Ministry of Education), Department of Radiotherapy, Peking University School of Oncology, Beijing Cancer Hospital, 52 Fu Cheng Lu, Beijing 100142Haidian District, PR China. ${ }^{4}$ Key Laboratory of Carcinogenesis and Translational Research (Ministry of Education), Department of Gastrointestinal Oncology, Peking University School of Oncology, Beijing Cancer Hospital, 52 Fu Cheng Lu, Beijing, Haidian District 100142, PR China.

Received: 30 January 2012 Accepted: 17 September 2012 Published: 29 October 2012

\section{References}

1. Lopez MJ, Barrios L: Evolution of pelvic exenteration. Surg Oncol Clin N Am 2005, 14:587-606.

2. National Comprehensive Cancer Network: Colon/Rectal Cancer Version v.2., http://www.nccn.org/professionals/physician_gls/PDF/colon/rectal.pdf.

3. Sharma A, Sharp DM, Walker LG, Monson JR: Colorectal MDTs: the team's perspective. Colorectal Dis 2008, 10:63-68. 
4. Bosset JF, Collette L, Calais G, Mineur L, Maingon P, Radosevic-Jelic L, Daban A, Bardet E, Beny A, Ollier JC: Chemotherapy with preoperative radiotherapy in rectal cancer. N Engl J Med 2006, 355:1114-1123.

5. Gérard JP, Conroy T, Bonnetain F, Bouché O, Chapet O, Closon-Dejardin MT, Untereiner M, Leduc B, Francois E, Maurel J, Seitz JF, Buecher B, Mackiewicz $R$, Ducreux M, Bedenne L: Preoperative radiotherapy with or without concurrent fluorouracil and leucovorin in T3-4 rectal cancers: results of FFCD 9203. J Clin Oncol 2006, 24:4620-4625.

doi:10.1186/1752-1947-6-369

Cite this article as: Zhan et al:: A multidisciplinary clinical treatment of locally advanced rectal cancer complicated with rectovesical fistula: a case report. Journal of Medical Case Reports 2012 6:369.

\section{Submit your next manuscript to BioMed Central and take full advantage of:}

- Convenient online submission

- Thorough peer review

- No space constraints or color figure charges

- Immediate publication on acceptance

- Inclusion in PubMed, CAS, Scopus and Google Scholar

- Research which is freely available for redistribution 